\title{
Simplicial complexes of whisker type
}

\author{
Mina Bigdeli* \\ Department of Mathematics \\ Institute for Advanced Studies in Basic Sciences \\ Zanjan, Iran \\ m.bigdelie@iasbs.ac.ir \\ Takayuki Hibi \\ Department of Pure and Applied Mathematics \\ Osaka University \\ Osaka, Japan \\ hibi@math.sci.osaka-u.ac.jp
}

\author{
Jürgen Herzog \\ Fachbereich Mathematik \\ Universität Duisburg-Essen \\ Essen, Germany \\ juergen.herzog@uni-essen.de \\ Antonio Macchia* \\ Fachbereich Mathematik und Informatik \\ Philipps-Universität Marburg \\ Marburg, Germany \\ macchia.antonello@gmail.com
}

Submitted: Dec 9, 2014; Accepted: Mar 12, 2015; Published: Mar 23, 2015

Mathematics Subject Classifications: Primary 13C15, 05E40, 05E45; Secondary 13D02

\begin{abstract}
Let $I \subset K\left[x_{1}, \ldots, x_{n}\right]$ be a zero-dimensional monomial ideal, and $\Delta(I)$ be the simplicial complex whose Stanley-Reisner ideal is the polarization of $I$. It follows from a result of Soleyman Jahan that $\Delta(I)$ is shellable. We give a new short proof of this fact by providing an explicit shelling. Moreover, we show that $\Delta(I)$ is even vertex decomposable. The ideal $L(I)$, which is defined to be the Stanley-Reisner ideal of the Alexander dual of $\Delta(I)$, has a linear resolution which is cellular and supported on a regular CW-complex. All powers of $L(I)$ have a linear resolution. We compute depth $L(I)^{k}$ and show that depth $L(I)^{k}=n$ for all $k \geqslant n$.
\end{abstract}

Keywords: depth function; linear quotients; vertex decomposable; whisker complexes; zero-dimensional ideals

\section{Introduction}

Graphs with whiskers have first been considered by Villarreal in [19]. They all share the nice property that they are Cohen-Macaulay. Various extensions of this concept and generalizations of his result have been considered in the literature, see $[2,8,13,18]$. The edge ideal of a whisker graph is obtained as the polarization of a monomial ideal $I \subset S$,

${ }^{*}$ The paper was written while the first and the fourth author were visiting the Department of Mathematics of University Duisburg-Essen. They want to express their thanks for the hospitality. 
where $S=K\left[x_{1}, \ldots, x_{n}\right]$ is the polynomial ring over a field $K, I$ is generated in degree 2 and $\operatorname{dim} S / I=0$. In particular, $I$ contains the squares $x_{1}^{2}, \ldots, x_{n}^{2}$. More generally, given a simplicial complex $\Gamma$, the whisker complex $W(\Gamma)$ is studied in [15]. Its facet ideal is the polarization of a monomial ideal in $S$ which contains all the $x_{i}^{2}$. In [15], Loiskekoski shows that the Stanley-Reisner ideal of the Alexander dual of the independence complex of $W(\Gamma)$ has a linear resolution, as well as its powers.

In the present paper we generalize the above mentioned results by considering the polarization of any monomial ideal $I \subset S$ with $\operatorname{dim} S / I=0$. The simplicial complex $\Theta(I)$, whose facet ideal coincides with the polarization $I^{\wp}$ of $I$, is called of whisker type - the whiskers being the simplices corresponding to the polarization of the pure powers contained in $I$. The independence complex of $\Theta(I)$, denoted $\Delta(I)$, is characterized by the property that the Stanley-Reisner ideal $I_{\Delta(I)}$ coincides with $I^{\wp}$. Note that $F \in \Delta(I)$ if and only if $F$ does not contain any facet of $\Theta(I)$.

Given an arbitrary monomial ideal $I \subset S$, a multicomplex is associated with $I$, as defined by Popescu and the second author in [11]. Soleyman Jahan defines in [17, Proposition 3.8] a bijection between the facets of the multicomplex given by $I$ and the facets of the simplicial complex associated with $I^{\wp}$. In Theorem 1 we present a short proof of this bijection when $\operatorname{dim} S / I=0$, by using multiplicity theory. This result allows us to describe in Corollary 2 the facets of $\Delta(I)$. By applying the Eagon-Reiner Theorem it is then shown in Corollary 3 that the ideal $L(I)$ has a linear resolution, where $L(I)$ is generated by the monomials $x_{1, a_{1}+1} \cdots x_{n, a_{n}+1}$ for which $x_{1}^{a_{1}} \cdots x_{n}^{a_{n}}$ is a monomial in $S$ not belonging to $I$.

In the case that $\operatorname{dim} S / I=0$, the case we consider here, the corresponding multicomplex is pretty clean, see [11]. Soleyman Jahan showed in [17, Theorem 4.3] that if $I$ defines a pretty clean multicomplex, then the simplicial complex associated with $I^{\wp}$ is clean, which, by a theorem of Dress [5], implies that the simplicial complex attached to $I^{\wp}$ is shellable. Applied to our situation it follows that $\Delta(I)$ is shellable. We give a direct proof of this fact by showing that $L(I)$ has linear quotients. This provides an explicit shelling of $\Delta(I)$, and as a side result we obtain a formula for the Betti numbers of $L(I)$ in terms of the $h$-vector of $S / I$, see Corollary 6 . We conclude Section 2 with Corollary 8 , where it is shown that the minimal graded free resolution of $L(I)$ is cellular and supported on a regular CW-complex. The proof is based on a result of Dochtermann and Mohammadi [4, Theorem 3.10], who showed that the minimal graded free resolution of any ideal with regular decomposition function, as defined in [12], have such nice cellular structure.

In Section 3 we show that $\Delta(I)$ is not only shellable but even vertex decomposable. This was already known for whisker graphs (see [3, Theorem 4.4]). Finally in Section 4 we prove that all powers of $L(I)$ have linear quotients, see Theorem 10. Analyzing the linear quotients, the depth function $f(k)=$ depth $S / L(I)^{k}$ can be computed. In Corollary 11 a formula for the depth function is given and $\lim _{k \rightarrow \infty}$ depth $S / L(I)^{k}$ is determined. 


\section{Independence complex of a whisker type simplicial complex}

Throughout this paper $S$ denotes the polynomial ring $K\left[x_{1}, \ldots, x_{n}\right]$ and $I \subset S$ a monomial ideal with $\operatorname{dim} S / I=0$, unless otherwise stated. The (finite) set of monomials in $S$ which belong to $S$ but not to $I$ will be denoted by $\operatorname{Mon}(S \backslash I)$. For an arbitrary monomial ideal $I$, we denote by $G(I)$ the unique minimal set of monomial generators of $I$. We will consider the polarization of $I$, denoted $I^{\wp}$. The polynomial ring in which $I^{\wp}$ is defined will be denoted by $S^{\wp}$.

In the following theorem (cf. [17, Proposition 3.8]) we determine the set $\operatorname{Min}\left(I^{\wp}\right)$ of minimal prime ideals of $I^{\wp}$.

Theorem 1. Let $I \subset S$ be a monomial ideal with $\operatorname{dim} S / I=0$. The map $\phi$ which assigns to each monomial $u=x_{1}^{a_{1}} \cdots x_{n}^{a_{n}} \in S \backslash I$ the monomial prime ideal $\phi(u)=$ $\left(x_{1, a_{1}+1}, \ldots, x_{n, a_{n}+1}\right) \subset S^{\wp}$, establishes a bijection between $\operatorname{Mon}(S \backslash I)$ and $\operatorname{Min}\left(I^{\wp}\right)$.

Proof. We first observe that $\phi(\operatorname{Mon}(S \backslash I)) \subset S^{\wp}$. Indeed, since $\operatorname{dim} S / I=0$, there exists for each $1 \leqslant i \leqslant n$ an integer $b_{i}>0$ such that $x_{i}^{b_{i}} \in I$ and $x_{i}^{b_{i}-1} \notin I$. It follows that $S^{\wp}$ is the polynomial ring in the variables $x_{i, 1}, \ldots, x_{i, b_{i}}$ with $1 \leqslant i \leqslant n$. Now let $u=x_{1}^{a_{1}} \cdots x_{n}^{a_{n}} \in \operatorname{Mon}(S \backslash I)$. Then $a_{i}<b_{i}$ for all $i$, and this implies that $\phi(u) \in S^{\wp}$.

Next we show that $\phi(\operatorname{Mon}(S \backslash I)) \subset \operatorname{Min}\left(I^{\wp}\right)$. In fact, let $u=x_{1}^{a_{1}} \cdots x_{n}^{a_{n}}$ be an element in $\operatorname{Mon}(S \backslash I)$, and let $v \in G(I)$. We claim that there exists an integer $i$ such that $x_{i, a_{i}+1}$ divides $v^{\wp}$, where $v^{\wp}$ is the polarization of $v$. From this claim it follows that $I^{\wp} \subset \phi(u)$. Since height $I^{\wp}=$ height $I=n$ and since height $\phi(u)=n$, we then see that $\phi(u)$ is in fact a minimal prime ideal of $I^{\wp}$.

Let $v=x_{1}^{b_{1}} \cdots x_{n}^{b_{n}}$. In order to prove the claim, note that $v^{\wp}=\prod_{i=1}^{n}\left(\prod_{j=1}^{b_{i}} x_{i, j}\right)$. Since $v$ does not divide $u$, there exists an integer $i$ such that $b_{i}>a_{i}$. Therefore, $x_{i, a_{i}+1}$ divides $v^{\wp}$, as desired.

Clearly, $\phi$ is injective. We will show that $|\operatorname{Mon}(S \backslash I)|=\left|\operatorname{Min}\left(I^{\wp}\right)\right|$. This will then imply that $\phi: \operatorname{Mon}(S \backslash I) \rightarrow \operatorname{Min}\left(I^{\wp}\right)$ is bijective. In order to see that these two sets have the same cardinality we observe that the multiplicity $e(S / I)$ of $S / I$ is equal to the length $\ell(S / I)$ of $S / I$, because $\operatorname{dim} S / I=0$, see [1, Corollary 4.7.11(b)]. Since $\ell(S / I)=$ $\operatorname{dim}_{K} S / I$ and since the elements of $\operatorname{Mon}(S \backslash I)$ form a $K$-basis of $S / I$, we see that $e(S / I)=\operatorname{dim}_{K} S / I=|\operatorname{Mon}(S \backslash I)|$. On the other hand, since $S / I$ is isomorphic to $S^{\wp} / I^{\wp}$ modulo a regular sequence of linear forms [9, Proposition 1.6.2], and since $S^{\wp} / I^{\wp}$ is reduced and equidimensional, [1, Corollary 4.7.8] implies that $e(S / I)=e\left(S^{\wp} / I^{\wp}\right)=\left|\operatorname{Min}\left(I^{\wp}\right)\right|$.

We denote by $\Delta(I)$ the simplicial complex whose Stanley-Reisner ideal is $I^{\wp}$. We view the variables $x_{i, j} \in S^{\wp}$ as the vertices of $\Delta(I)$. As an immediate consequence of Theorem 1 we obtain

Corollary 2. Let $\mathcal{S}$ be the set of variables of $S^{\wp}$. Then $F \subset \mathcal{S}$ is a facet of $\Delta(I)$ if and only if there exists $x_{1}^{a_{1}} \cdots x_{n}^{a_{n}} \in \operatorname{Mon}(S \backslash I)$ such that

$$
F=\mathcal{S} \backslash\left\{x_{1, a_{1}+1}, \ldots, x_{n, a_{n}+1}\right\} .
$$


Since $\Delta(I)$ is Cohen-Macaulay, the Eagon-Reiner Theorem [6] (see also [9, Theorem 8.1.9]) implies that $I_{\Delta(I) \vee}$ has a linear resolution. Here $\Delta(I)^{\vee}$ denotes the Alexander dual of $\Delta(I)$. Recall that, if $\Delta$ is an arbitrary simplicial complex on the vertex set $[n]=\{1, \ldots, n\}$ and $I_{\Delta}=\bigcap_{F} P_{F}$ where $P_{F}=\left(x_{i}: i \in F\right)$, then $I_{\Delta} \vee$ is generated by the monomials $u_{F}$ where $u_{F}=\prod_{i \in F} x_{i}$. These facts applied to our case yield

Corollary 3. The ideal $L(I)$ generated by the monomials $x_{1, a_{1}+1} \cdots x_{n, a_{n}+1}$, with $x_{1}^{a_{1}} \cdots x_{n}^{a_{n}} \in \operatorname{Mon}(S \backslash I)$, has a linear resolution.

In the following we consider the special case that $x_{i}^{2} \in I$ for all $i$. In that case all other generators of $I$ are square-free. In simplified notation, the polarization $I^{\wp}$ of $I$ is generated by the square-free monomials in $I$ and by the monomials $x_{i} y_{i}$ for $i=1, \ldots, n$.

Let $\Gamma$ be the simplicial complex with $I(\Gamma)=J$ and $W(\Gamma)$ be the simplicial complex with $I(W(\Gamma))=\left(J, x_{1} y_{1}, \ldots, x_{n} y_{n}\right)$. The edges of $W(\Gamma)$ corresponding to the $x_{i} y_{i}$ are called the whiskers of $W(\Gamma)$ and $W(\Gamma)$ is called the whisker complex of $\Gamma$.

Given a simplicial complex $\Sigma$, the independence complex $\Lambda$ of $\Sigma$ is the simplicial complex such that $I_{\Lambda}=I(\Sigma)$. Notice that $F \in \Lambda$ if and only if no face of $\Sigma$ is contained in $F$.

Corollary 4. Let $\Gamma$ be a simplicial complex on the vertex set $[n], I^{\prime}=I(\Gamma)$ the facet ideal of $\Gamma$ and $W(\Gamma)$ its whisker complex. Let $I=\left(I^{\prime}, x_{1}^{2}, x_{2}^{2}, \ldots, x_{n}^{2}\right)$. Then $\Delta(I)$ is the independence complex of $W(\Gamma)$ and $L(I)$ is generated by the monomials $\prod_{i \in[n] \backslash F} x_{i} \prod_{i \in F} y_{i}$ with $F \in \Delta$, where $\Delta$ is the independence complex of $\Gamma$.

\section{$3 \quad$ Linear quotients}

Let $I \subset S$ be a monomial ideal with $\operatorname{dim} S / I=0$. The main purpose of this section is to show that $L(I)$ not only has a linear resolution, but even has linear quotients.

Theorem 5. The ideal $L(I)$ has linear quotients.

Proof. Let $u, v \in G(L(I)), u=x_{1, a_{1}+1} \cdots x_{n, a_{n}+1}$ and $v=x_{1, b_{1}+1} \cdots x_{n, b_{n}+1}$. We set $u \leqslant v$ if $a_{i} \leqslant b_{i}$ for all $i$, and extend this partial order to a total order on $G(L(I))$. We claim that, with respect to this total order of the monomial generators of $L(I)$, the ideal $L(I)$ has linear quotients. Indeed, let $x_{1, a_{1}+1} \cdots x_{n, a_{n}+1}$ be the largest element in $G(L(I))$. Then $u=x_{1}^{a_{1}} \cdots x_{n}^{a_{n}} \in \operatorname{Mon}(S \backslash I)$ and $x_{i} u \in I$ for all $i$. Set $I^{\prime}=I+(u)$. Then the polarization $\left(I^{\prime}\right)^{\wp}$ of $I^{\prime}$ is equal to $I_{\Delta\left(I^{\prime}\right)}$. Notice that $L\left(I^{\prime}\right) \subset L(I)$ and $\ell\left(S / I^{\prime}\right)<\ell(S / I)$. In particular, $L(I)=\left(L\left(I^{\prime}\right), x_{1, a_{1}+1} \cdots x_{n, a_{n}+1}\right)$. Arguing by induction on the length, we may assume that $L\left(I^{\prime}\right)$ has linear quotients. Thus we just need to compute the colon ideal $Q=L\left(I^{\prime}\right): x_{1, a_{1}+1} \cdots x_{n, a_{n}+1}$. We claim that

$$
Q=\left(x_{1,1}, x_{1,2}, \ldots, x_{1, a_{1}}, x_{2,1}, \ldots, x_{2, a_{2}}, \ldots, x_{n, 1}, \ldots, x_{n, a_{n}}\right) .
$$

Suppose that $j \in\left\{1, \ldots, a_{i}\right\}$ for some $i$. Then $x_{1}^{a_{1}} \cdots x_{i}^{j-1} \cdots x_{n}^{a_{n}} \in \operatorname{Mon}(S \backslash I)$ and

$$
\phi\left(x_{1}^{a_{1}} \cdots x_{i}^{j-1} \cdots x_{n}^{a_{n}}\right)=x_{1, a_{1}+1} \cdots x_{i, j} \ldots x_{n, a_{n}+1} \in L\left(I^{\prime}\right) .
$$


It follows that $x_{i, j} \in Q$.

On the other hand, the elements $v / \operatorname{gcd}\left(v, x_{1, a_{1}+1} \cdots x_{n, a_{n}+1}\right)$ with $v \in G\left(L\left(I^{\prime}\right)\right)$ generate $Q$, see for example [9, Proposition 1.2.2]. In fact, let $v \in G\left(L\left(I^{\prime}\right)\right)$. Then $v=$ $x_{1, c_{1}+1} \cdots x_{n, c_{n}+1}$ and $x_{1}^{c_{1}} \cdots x_{n}^{c_{n}} \in \operatorname{Mon}\left(S \backslash I^{\prime}\right)$. There exists $i$ such that $c_{i}<a_{i}$ because $x_{i} u \in I$ for all $i$. Hence $x_{i, c_{i}+1}$ does not divide $x_{1, a_{1}+1} \cdots x_{n, a_{n}+1}$, and therefore $x_{i, c_{i}+1}$ divides $v / \operatorname{gcd}\left(v, x_{1, a_{1}+1} \cdots x_{n, a_{n}+1}\right)$. Since $c_{i}+1 \leqslant a_{i}$, the desired conclusion follows.

Corollary 6. For every $i \geqslant 0$,

$$
\beta_{i}\left(S^{\wp} / L(I)\right)=\sum_{j \geqslant 0} h_{j}\left(\begin{array}{c}
j \\
i-1
\end{array}\right),
$$

where $h_{j}=h_{j}(S / I)$ is the $j$-th component of the $h$-vector of $S / I$. In particular, proj $\operatorname{dim} S^{\wp} / L(I)=\max \{\operatorname{deg} u: u \in \operatorname{Mon}(S \backslash I)\}+1$.

Proof. As in the previous proof, let $u=x_{1}^{a_{1}} \cdots x_{n}^{a_{n}} \in \operatorname{Mon}(S \backslash I)$ with $x_{i} u \in I$ for all $i$. Set $I^{\prime}=I+(u)$, and consider the short exact sequence

$$
0 \rightarrow L(I) / L\left(I^{\prime}\right) \rightarrow S^{\wp} / L\left(I^{\prime}\right) \rightarrow S^{\wp} / L(I) \rightarrow 0 .
$$

Notice that $L(I) / L\left(I^{\prime}\right) \cong S^{\wp} / Q(-n)$ with $Q$ as in (1). Hence its minimal free resolution is the Koszul complex $\mathbb{K}$ on the variables $x_{i, j}$ with $x_{i, j} \in G(Q)$. Thus the minimal free resolution of $S^{\wp} / L(I)$ can be obtained as a mapping cone of $\mathbb{K}$ and the minimal free resolution of $S^{\wp} / L\left(I^{\prime}\right)$. Therefore $\beta_{0}\left(S^{\wp} / L(I)\right)=\beta_{0}\left(S^{\wp} / L\left(I^{\prime}\right)\right)$, and for $i \geqslant 1$ we obtain

$$
\begin{aligned}
\beta_{i}\left(S^{\wp} / L(I)\right) & =\beta_{i}\left(S^{\wp} / L\left(I^{\prime}\right)\right)+\operatorname{rank}\left(K_{i-1}\right)=\beta_{i}\left(S^{\wp} / L\left(I^{\prime}\right)\right)+\left(\begin{array}{c}
\operatorname{deg} u \\
i-1
\end{array}\right) \\
& =\sum_{u \in \operatorname{Mon}(S \backslash I)}\left(\begin{array}{c}
\operatorname{deg} u \\
i-1
\end{array}\right)=\sum_{j \geqslant 0} h_{j}\left(\begin{array}{c}
j \\
i-1
\end{array}\right) .
\end{aligned}
$$

It is easily seen that the geometric realization of $\Delta(I)$ is a sphere if $I$ is a complete intersection, and a ball otherwise. Both topological spaces admit shellable triangulations, though in general not all triangulations of these spaces are shellable, see [16] and [14]. However, due to Theorem 5 we have

Corollary 7. The simplicial complex $\Delta(I)$ is shellable.

As a further consequence of Theorem 5 we have

Corollary 8. The graded minimal free resolution of $L(I)$ is cellular and supported on a regular $C W$-complex. 
Proof. Since $L(I)$ has linear quotients we may apply [4, Theorem 3.10] and only need to show that $L(I)$ admits a regular decomposition function. In order to explain this, let $J=\left(u_{1}, \ldots, u_{m}\right)$ be an ideal with linear quotients with respect to the given order of the generators. The decomposition function of $J$ (with respect to the given order of the generators of $J$ ) is the map $b: \operatorname{Mon}(J) \rightarrow G(J)$ with $b(u)=u_{j}$, where $j$ is the smallest number such that $u \in\left(u_{1}, \ldots, u_{j}\right)$. For each $u_{j} \in G(J)$, let $\operatorname{set}\left(u_{j}\right)$ be the set of all $x_{i}$ such that $x_{i} u_{j} \in\left(u_{1}, \ldots, u_{j-1}\right)$. According to [12], the decomposition function $b$ is called regular, if $\operatorname{set}\left(b\left(x_{i} u_{j}\right)\right) \subset \operatorname{set}\left(u_{j}\right)$ for all $u_{j} \in G(J)$ and all $x_{i} \in \operatorname{set}\left(u_{j}\right)$.

Now let $u \in G(L(I)), u=x_{1, a_{1}+1} \cdots x_{n, a_{n}+1}$. By (1) we have

$$
\operatorname{set}(u)=\left\{x_{1,1}, x_{1,2}, \ldots, x_{1, a_{1}}, x_{2,1}, \ldots, x_{2, a_{2}}, \ldots, x_{n, 1}, \ldots, x_{n, a_{n}}\right\} .
$$

Let $x_{i, j} \in \operatorname{set}(u)$. Then $b\left(x_{i, j} u\right)=x_{i, j}\left(u / x_{i, a_{i}+1}\right)$, and so

$$
\operatorname{set}\left(b\left(x_{i, j} u\right)\right)=\operatorname{set}(u) \backslash\left\{x_{i, j+1}, \ldots, x_{i, a_{i}}\right\} \subset \operatorname{set}(u),
$$

as desired.

\section{Vertex decomposability}

In [3, Theorem 4.4] it was shown that for any graph, the independence complex of its whisker graph is vertex decomposable. Here we extend this result by showing that $\Delta(I)$ is vertex decomposable for any monomial ideal $I$ with $\operatorname{dim} S / I=0$. Recall that a simplicial complex $\Delta$ is called vertex decomposable if $\Delta$ is a simplex, or $\Delta$ contains a vertex $v$ such that

(i) any facet of $\operatorname{del}_{\Delta}(v)$ is a facet of $\Delta$, and

(ii) both $\operatorname{del}_{\Delta}(v)$ and $\operatorname{link}_{\Delta}(v)$ are vertex decomposable.

Here $\operatorname{link}_{\Delta}(v)=\{G \in \Delta: v \notin G$ and $G \cup\{v\} \in \Delta\}$ is the link of $v$ in $\Delta$ and $\operatorname{del}_{\Delta}(v)=$ $\{G \in \Delta: v \notin G\}$ is the deletion of $v$ from $\Delta$.

A vertex $v$ which satisfies condition (i) is called a shedding vertex of $\Delta$.

For the proof of the next result we observe the following fact: let $\Delta$ be a simplicial complex, $\mathcal{F}(\Delta)$ the set of its facets and $v$ a vertex not belonging to $\Delta$. The cone of $v$ over $\Delta$, denoted by $v * \Delta$, is the simplicial complex whose set of facets is $\mathcal{F}(v * \Delta)=\{\{v\} \cup F$ : $F \in \mathcal{F}(\Delta)\}$. If $\Delta$ is vertex decomposable, then $v * \Delta$ is again vertex decomposable (with respect to the same shedding vertex).

Theorem 9. Let $I$ be a monomial ideal in $S=K\left[x_{1}, \ldots, x_{n}\right]$ with $\operatorname{dim} S / I=0$. Then $\Delta(I)$ is vertex decomposable.

Proof. By assumption, for each $1 \leqslant i \leqslant n$ there exists $b_{i} \geqslant 1$ such that $x_{i}^{b_{i}} \in G(I)$. Then $\Delta(I)$ is a simplicial complex on $\mathcal{S}=\left\{x_{1,1}, \ldots, x_{1, b_{1}}, \ldots, x_{n, 1}, \ldots, x_{n, b_{n}}\right\}$. We proceed by 
induction on $\sum_{i=1}^{n} b_{i}$. If $\sum_{i=1}^{n} b_{i}=n$, then $I=\left(x_{1}, \ldots, x_{n}\right)$, which is a trivial case. Suppose that $\sum_{i=1}^{n} b_{i}>n$. Hence we may assume $b_{n}>1$.

We first show that the vertex $x_{n, 1}$ is a shedding vertex of $\Delta(I)$. Clearly,

$$
\operatorname{del}_{\Delta(I)}\left(x_{n, 1}\right)=\left\{F: F \in \Delta(I), x_{n, 1} \notin F\right\} \cup\left\{F \backslash\left\{x_{n, 1}\right\}: F \in \Delta(I), x_{n, 1} \in F\right\} .
$$

Obviously, any facet of $\operatorname{del}_{\Delta(I)}\left(x_{n, 1}\right)$ with $x_{n, 1} \notin F$ is a facet of $\Delta(I)$. On the other hand, if we consider $F \backslash\left\{x_{n, 1}\right\}$ with $F \in \mathcal{F}(\Delta(I))$ and $x_{n, 1} \in F$, then $F \backslash\left\{x_{n, 1}\right\}$ is not a facet of $\operatorname{del}_{\Delta(I)}\left(x_{n, 1}\right)$. Indeed, since $F \in \mathcal{F}(\Delta(I))$, there exists $u \in \operatorname{Mon}(S \backslash I)$ such that $\phi(u)=P_{\mathcal{S} \backslash F}$. Let $t$ be the largest integer such that $x_{n}^{t}$ divides $u$. Then $x_{n, t+1} \in P_{\mathcal{S} \backslash F}$ and so $x_{n, j} \in F$ for all $j \neq t+1$. Since $x_{n, 1} \in F$, we have $t+1 \neq$ 1. Let $u^{\prime}=u / x_{n}^{t}$. Then $u^{\prime} \in \operatorname{Mon}(S \backslash I)$ and $\phi\left(u^{\prime}\right)=P_{\left((\mathcal{S} \backslash F) \backslash\left\{x_{n, t+1}\right\}\right) \cup\left\{x_{n, 1}\right\} \text {. Thus }}$ $G=\left(F \backslash\left\{x_{n, 1}\right\}\right) \cup\left\{x_{n, t+1}\right\} \in \mathcal{F}(\Delta(I))$. Since $G \in \operatorname{del}_{\Delta(I)}\left(x_{n, 1}\right)$, the claim follows. Consequently, $\mathcal{F}\left(\operatorname{del}_{\Delta(I)}\left(x_{n, 1}\right)\right)=\left\{F: F \in \mathcal{F}(\Delta(I)), x_{n, 1} \notin F\right\}$ which implies that $x_{n, 1}$ is a shedding vertex of $\Delta(I)$.

We now prove that $\operatorname{del}_{\Delta(I)}\left(x_{n, 1}\right)$ and $\operatorname{link}_{\Delta(I)}\left(x_{n, 1}\right)$ are vertex decomposable.

First we consider $\operatorname{del}_{\Delta(I)}\left(x_{n, 1}\right)$. Let $J_{1}$ be the ideal in $S$ with $\operatorname{Mon}\left(S \backslash J_{1}\right)=\{u$ : $u \in \operatorname{Mon}(S \backslash I), x_{n}$ does not divide $\left.u\right\}$. Then $\Delta\left(J_{1}\right)$ is a simplicial complex on $\mathcal{S} \backslash$ $\left\{x_{n, 1}, \ldots, x_{n, b_{n}}\right\}$. By using Corollary 2 we see that

$$
\operatorname{del}_{\Delta(I)}\left(x_{n, 1}\right)=x_{n, b_{n}} *\left(x_{n, b_{n}-1} *\left(\cdots *\left(x_{n, 2} * \Delta\left(J_{1}\right)\right)\right)\right) .
$$

Our induction hypothesis implies that $\Delta\left(J_{1}\right)$ is vertex decomposable, hence $\operatorname{del}_{\Delta(I)}\left(x_{n, 1}\right)$ is vertex decomposable.

As for $\operatorname{link}_{\Delta(I)}\left(x_{n, 1}\right)$, let $\Gamma$ be the simplicial complex whose faces are obtained from the faces of $\operatorname{link}_{\Delta(I)}\left(x_{n, 1}\right)$ as follows: for every $F \in \operatorname{link}_{\Delta(I)}\left(x_{n, 1}\right)$, we replace each $x_{n, j} \in F$ by $x_{n, j-1}$. Hence $\Gamma$ is a simplicial complex on $\mathcal{S} \backslash\left\{x_{n, b_{n}}\right\}$ and $\Gamma \cong \operatorname{link}_{\Delta(I)}\left(x_{n, 1}\right)$. Let $J_{2}$ be the monomial ideal in $S$ such that $\operatorname{Mon}\left(S \backslash J_{2}\right)=\left\{u / x_{n}: u \in \operatorname{Mon}(S \backslash I), x_{n}\right.$ divides $\left.u\right\}$. Then Corollary 2 implies that $\Gamma=\Delta\left(J_{2}\right)$, which is vertex decomposable by induction hypothesis. It follows that $\operatorname{link}_{\Delta(I)}\left(x_{n, 1}\right)$ is vertex decomposable, as desired.

\section{Powers}

In this section we study the powers of $L(I)$. The main result is

Theorem 10. Let $I \subset S$ be a monomial ideal with $\operatorname{dim} S / I=0$. Then $L(I)^{k}$ has linear quotients for all $k$. In particular, all powers of $L(I)$ have a linear resolution.

Proof. Any $u \in L(I)^{k}$ can be written in the form $u=u_{1}^{\prime} u_{2}^{\prime} \cdots u_{n}^{\prime}$, where $u_{i}^{\prime}=$ $x_{i, j(i)_{1}} x_{i, j(i)_{2}} \cdots x_{i, j(i)_{k}}$ for $i=1, \ldots, n$ with $j(i)_{1} \leqslant j(i)_{2} \leqslant \cdots \leqslant j(i)_{k}$. We define a partial order on $G\left(L(I)^{k}\right)$ by setting $v \leqslant u$, if, with respect to the lexicographical order, $u_{i}^{\prime} \leqslant v_{i}^{\prime}$ for all $i$, and we extend this partial order to a total order on the set of monomial generators of $L(I)^{k}$.

Now let $v, u \in L(I)^{k}$ with $v<u$. We need to show that there exists $w \in L(I)^{k}$ with $w<u$ such that $w / \operatorname{gcd}(w, u)$ is of degree 1 and $\operatorname{such}$ that $w / \operatorname{gcd}(w, u)$ divides 
$v / \operatorname{gcd}(v, u)$. Indeed, since $v<u$, there exists $i$ such that $u_{i}^{\prime}<v_{i}^{\prime}$ in the lexicographical order. Thus if $v_{i}^{\prime}=x_{i, j^{\prime}(i)_{1}} x_{i, j^{\prime}(i)_{2}} \cdots x_{i, j^{\prime}(i)_{k}}$ with $j^{\prime}(i)_{1} \leqslant j^{\prime}(i)_{2} \leqslant \cdots \leqslant j^{\prime}(i)_{k}$, then there exists $\ell$ such that $j^{\prime}(i)_{s}=j(i)_{s}$ for $s<\ell$ and $j^{\prime}(i)_{\ell}<j(i)_{\ell}$. We let $w=w_{1}^{\prime} w_{2}^{\prime} \cdots w_{n}^{\prime}$ with $w_{t}^{\prime}=u_{t}^{\prime}$ for $t \neq i$ and $w_{i}^{\prime}=x_{i, j^{\prime}\left(i_{\ell}\right.}\left(u_{i}^{\prime} / x_{i, j(i)_{\ell}}\right)$.

It is clear that $w<u$. Furthermore, $w \in G\left(L(I)^{k}\right)$. In fact, $u=u_{1} \cdots u_{k}$ with $u_{i} \in$ $L(I)$, and $x_{i, j(i)_{\ell}}$ divides one of these factors, say it divides $u_{r}$. Then $\bar{u}_{r}=x_{i, j^{\prime}\left(i_{\ell}\right.}\left(u_{r} / x_{i, j(i)_{\ell}}\right)$ belongs to $L(I)$ since $j^{\prime}(i)_{\ell}<j(i)_{\ell}$, and hence $w=u_{1} \cdots \bar{u}_{r} \cdots u_{k}$ belongs to $G\left(L(I)^{k}\right)$. Note further that $w / \operatorname{gcd}(w, u)=x_{i, j^{\prime}(i)_{\ell}}$ and that $x_{i, j^{\prime}(i)_{\ell}}$ divides $v / \operatorname{gcd}(v, u)$. This completes the proof.

Corollary 11. For $i=1, \ldots, n$, let $b_{i}$ be the smallest integer such that $x_{i}^{b_{i}} \in I$. Then $\operatorname{depth} S^{\wp} / L(I)^{k}=\sum_{i=1}^{n} b_{i}-\max \left\{\operatorname{deg}\left(\operatorname{lcm}\left(u_{1}, \ldots, u_{k}\right)\right): u_{1}, \ldots, u_{k} \in \operatorname{Mon}(S \backslash I)\right\}-1$. In particular, depth $S^{\wp} / L(I)^{k}=n-1$ for all $k \geqslant n$, and

$$
\operatorname{depth} S^{\wp} / L(I)^{k}>\operatorname{depth} S^{\wp} / L(I)^{k+1},
$$

as long as depth $S^{\wp} / L(I)^{k}>n-1$.

Proof. In general, let $J \subset K\left[x_{1}, \ldots, x_{n}\right]$ be a graded ideal generated by a sequence $f_{1}, \ldots, f_{s}$ with linear quotients, and denote by $q_{j}(J)$ the minimal number of linear forms generating the ideal $\left(f_{1}, f_{2}, \ldots, f_{j-1}\right): f_{j}$. Then depth $K\left[x_{1}, \ldots, x_{n}\right] / J=n-q(J)-1$, where $q(J)=\max \left\{q_{j}(J): 2 \leqslant j \leqslant s\right\}$, see [10, Formula (1)].

We apply this formula to $S^{\wp} / L(I)^{k}$. Since the Krull dimension of $S^{\wp}$ is equal to $\sum_{i=1}^{n} b_{i}$, it remains to be shown that

$$
q\left(L(I)^{k}\right)=\max \left\{\operatorname{deg}\left(\operatorname{lcm}\left(u_{1}, u_{2}, \ldots, u_{k}\right)\right): u_{1}, u_{2}, \ldots, u_{k} \in \operatorname{Mon}(S \backslash I)\right\} .
$$

To see this, let $u_{1}, u_{2}, \ldots, u_{k} \in \operatorname{Mon}(S \backslash I)$ where $u_{j}=x_{1}^{a_{1}(j)} \cdots x_{n}^{a_{n}(j)}$ for $j=1, \ldots, k$. Then $u=u_{1}^{\prime} u_{2}^{\prime} \cdots u_{n}^{\prime}$ with $u_{i}^{\prime}=\prod_{j=1}^{k} x_{i, a_{i}(j)+1}$ is a generator of $L(I)^{k}$. We may assume that $u$ is the $j$-th element in the given total order of the elements of $G\left(L(I)^{k}\right)$. As shown in the proof of Theorem $10, q_{j}\left(L(I)^{k}\right)$ is the cardinality of the set

$$
\left\{x_{1,1}, \ldots, x_{1, c_{1}}, x_{2,1}, \ldots, x_{2, c_{2}}, \ldots, x_{n, 1}, \ldots, x_{n, c_{n}}\right\},
$$

where $c_{i}=\max \left\{a_{i}(1), \ldots, a_{i}(k)\right\}$ for $i=1, \ldots, n$. If follows that

$$
q_{j}\left(L(I)^{k}\right)=\operatorname{deg}\left(\operatorname{lcm}\left(u_{1}, u_{2}, \ldots, u_{k}\right)\right),
$$

and hence equation (2) follows.

Suppose now that $k \geqslant n$. Then we may choose $u_{i}=x_{i}^{b_{i}-1}$ for $i=1, \ldots, n$ and $u_{i} \in \operatorname{Mon}(S \backslash I)$ arbitrary for $i>n$, and obtain $\operatorname{deg}\left(\operatorname{lcm}\left(u_{1}, u_{2}, \ldots u_{k}\right)\right)=\sum_{i=1}^{n}\left(b_{i}-1\right)=$ $\sum_{i=1}^{n} b_{i}-n$. Since this is the largest possible least common multiple of sequences of elements of $\operatorname{Mon}(S \backslash I)$, it follows that depth $S^{\wp} / L(I)^{k}=n-1$ for all $k \geqslant n$. 
Finally, suppose that depth $S^{\wp} / L(I)^{k}>n-1$. Then the formula for depth $S^{\wp} / L(I)^{k}$ implies that $\max \left\{\operatorname{deg}\left(\operatorname{lcm}\left(u_{1}, \ldots, u_{k}\right)\right): u_{1}, \ldots, u_{k} \in \operatorname{Mon}(S \backslash I)\right\}<\sum_{i=1}^{n}\left(b_{i}-1\right)$.

Let $x_{1}^{a_{1}} \cdots x_{n}^{a_{n}}=\operatorname{lcm}\left(u_{1}, \ldots, u_{k}\right)$ attain this maximal degree. Since $\sum_{i=1}^{n} a_{i}<$ $\sum_{i=1}^{n}\left(b_{i}-1\right)$, there exists an index $i$ such that $a_{i}<b_{i}-1$. Let $u_{k+1}=x_{i}^{b_{i}-1}$. Then $\operatorname{deg}\left(\operatorname{lcm}\left(u_{1}, \ldots, u_{k}, u_{k+1}\right)\right)>\operatorname{deg}\left(\operatorname{lcm}\left(u_{1}, \ldots, u_{k}\right)\right)$. Consequently, $\operatorname{depth} S^{\wp} / L(I)^{k}>$ depth $S^{\wp} / L(I)^{k+1}$, as desired.

\section{References}

[1] W. Bruns, J. Herzog. Cohen-Macaulay rings, Revised Edition. Cambridge University Press, Cambridge, 1996.

[2] D. Cook II, U. Nagel. Cohen-Macaulay graphs and face vectors of flag complexes. SIAM J. Discrete Math., 26:89-101, 2012.

[3] A. Dochtermann, A. Engström. Algebraic properties of edge ideals via combinatorial topology. Electron. J. Combin., 16(2):\#R2, 2009.

[4] A. Dochtermann, F. Mohammadi. Cellular resolutions from mapping cones. J. Combin. Theory Ser. A, 128:180-206, 2014.

[5] A. Dress. A new algebraic criterion for shellability. Beitr. Algebra Geom., 34:45-55, 1993.

[6] J. A. Eagon, V. Reiner. Resolutions of Stanley-Reisner rings and Alexander duality. J. Pure Appl. Algebra, 130:265-275, 1998.

[7] C. A. Francisco, H. T. Hà. Whiskers and sequentially Cohen-Macaulay graphs. J. Combin. Theory Ser. A, 115:304-316, 2008.

[8] S. Faridi. Cohen-Macaulay properties of square-free monomial ideals. J. Combin. Theory Ser. A, 109:299-329, 2005.

[9] J. Herzog, T. Hibi. Monomial Ideals. Graduate Text in Mathematics, Springer, 2011.

[10] J. Herzog, T. Hibi. The depth of powers of an ideal. J. Algebra, 291:534-550, 2005.

[11] J. Herzog, D. Popescu. Finite filtrations of modules and shellable multicomplexes. Manuscripta Math., 121:385-410, 2006.

[12] J. Herzog, Y. Takayama. Resolutions by mapping cones. Homology Homotopy Appl., 4(2):277-294, 2002.

[13] T. Hibi, A. Higashitani, K. Kimura, A. B. O'Keefe. Algebraic study on CameronWalker graphs. J. Algebra, 422:257-269, 2015.

[14] W. B. R. Lickorish. Unshellable triangulations of spheres. European J. Combin., 12:527-530, 1991.

[15] L. Loiskekoski. Resolutions and associated primes of powers of ideals. Master Thesis, Aalto University, School of Science, 2013.

[16] M. E. Rudin. An unshellable triangulation of a tetrahedron. Bull. Amer. Math. Soc., 64:90-91, 1958. 
[17] A. Soleyman Jahan. Prime filtrations of monomial ideals and polarizations. $J$. Algebra, 312:1011-1032, 2007.

[18] A. Van Tuyl, R. H. Villarreal. Shellable graphs and sequentially Cohen-Macaulay bipartite graphs. J. Combin. Theory Ser. A, 115:799-814, 2008.

[19] R. H. Villarreal. Cohen-Macaulay graphs. Manuscripta Math., 66:277-293, 1990. 